\title{
Prediction of coating geometry: theory and experiment
}

\author{
O. Nenadl, V. Ocelík, A. Palavra \& J. Th. M. De Hosson \\ Materials Innovation Institute, Department of Applied Physics, \\ University of Groningen, The Netherlands
}

\begin{abstract}
In this contribution we present a comparison between laser clad experiments and model predictions of the entire geometry of laser deposited multi-layered coatings. Recently we have shown that the recursive model describing the geometry of laser clad coatings combined with experimental track characteristics leads to specific functions describing the geometry of coatings formed by overlap of individual tracks depending on the processing parameters. The recursive model provides an adequate description of the whole geometry of the coating from the Height $H$ and width $w$ of a single laser track for any overlap ratio $O R$. We have shown that the height and width of a single track are well correlated with the main laser cladding processing parameters for both coaxial and side cladding set-ups.

Combining these two approaches leads to a prediction of the complete geometry of laser clad coatings from a number of basic processing parameters. These parameters are: Feeding rate $F$, Laser beam scanning speed $S$ and overlap ratio $O R$. The functions that describe the height and waviness of the final coating are the same for both coaxial and side cladding set-ups. These model predictions lead to very simple, yet very accurate predictions of the entire geometry of the laser deposited layers.

Keywords: laser cladding, laser clad geometry, laser additive manufacturing, material processing, modelling.
\end{abstract}

\section{Introduction}

In laser cladding thick protective coatings can be deposited on low-cost substrates. The process consists of an addition of a material via cladding on the surface of a substrate, where the heat source is a high power laser beam. This 
results in a clad layer with a typical thickness of $0.5-2 \mathrm{~mm}$ in a single step. Thicker layers can be achieved by building additional layers on top of the original coating. In our studies we use powder as a cladding material as this type of laser cladding leads to high quality, thick coatings with metallurgic bonds and minimal heat input into the work piece [1].

A complete description of the cladding process is complicated due to interaction of many complex physical phenomena and depends on additional parameters such as laser beam spot size, laser beam energy distribution, carrier and shielding gas used, how exactly the powder is fed, geometry of the substrate etc. Despite this, we can identify three key parameters that can be controlled easily in the experiment and strongly determine the final coating. These are: laser power $P(W)$, laser beam scanning speed $S(\mathrm{~mm} / \mathrm{s})$ and powder feeding rate $F$ $(\mathrm{mg} / \mathrm{s})$. We refer to these as processing parameters. Our past work has shown particular statistical relationships between these processing parameters and basic geometrical characteristics of the laser track for coaxial [2] and side laser cladding nozzles [3].

The laser cladding treatment can be considered as process that is based on the transfer of energy from the laser beam to the substrate and the powder and the transfer of mass between the powder flow and the molten surface. These mechanisms depend directly on the processing parameters. It has been demonstrated previously [4] that each of these two mechanisms relates to a specific combination of the processing parameters and hence we can define the so called combined parameters. These quantities can be expressed in the following way: the amount of powder delivered per unit length of the laser track $F / S$ and the total heat input per unit length of the laser track $P / S$. It has been shown experimentally that over a wide range of processing parameters the clad height, $H$, depends linearly on the $F / S$ parameter with the laser power having a minimal effect. Similarly the width, $w$, of the laser track linearly depends on $P / \sqrt{ } S$ and the clad area, $A_{c}$, is controlled by the $\sqrt{ } P^{*} F / S$ parameter. These empirical dependencies were observed for both, side and coaxial cladding setups with high values of the correlation coefficient $(\mathrm{R}>0.9)$ for cladding of $\mathrm{Ni}$ and Co based coatings on iron base substrates $[2,3]$ and have been confirmed theoretically and experimentally for other materials [5-8].

Recently we have designed a recursive model for prediction of a profile of coatings created by overlap of individual laser tracks [9] based on simplified physical assumptions. Defining the overlap ratio, $O R$, i.e. relating track width $w$ with distance $D$ between the centres of neighbouring tracks:

$$
O R=(w-D) / w
$$

The assumptions in the model are:

1. The width of the track is controlled by the dimensions of the laser beam (in case of laser cladding by the width of laser beam) and stays constant during the track overlap;

2. The character of the track profile shape is controlled by physical parameters such as viscosity, surface energy of the melt, gravitational force, etc. and is not changed by overlap; 
3. The amount of the clad material is constant during successive cladding tracks.

Mathematically the recursive model is formulated in the following fashion: The width of the single track $w$ is defined by the distance between points $A_{l}$ and $B_{l}$ on a horizontal axis, Fig. 1 . The profile of the first track is given by a known function $F_{l}$. A hypothetical position of the second, 'shifted' laser track with the same profile as $F_{1}$ is marked by a dashed profile between points $A_{2}$ and $B_{2}$. The overlap ratio $O R$ is defined as the distance between points $A_{2}$ and $B_{1}$ divided by the track width $w$. The real profile of the second overlapped track $F_{2}$ has to be found on the base of function $F_{1}$ and physical assumptions made above. Similarly, all other profiles are calculated recursively on the base of the previous one.

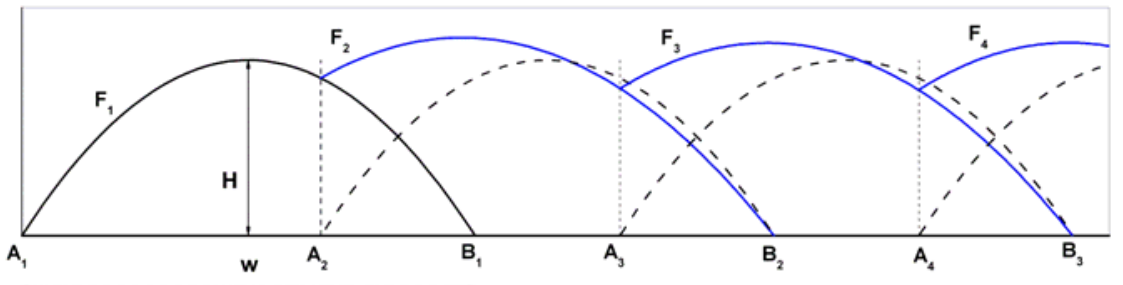

Figure 1: $\quad$ Schematic representation of the recursive model [9].

It is assumed that function $F_{l}$ represents the integration over an interval $\left(A_{l}\right.$, $\left.B_{1}\right)$ and that this function is equal to zero outside of this interval. Let $z=(1-O R)$. The left point and the right point of $n$-th track are: $A_{i}=w z(i-1), B_{i}=w+w z(i-1)$, $i=1,2 \ldots n$. Profile of $F_{i}$ starts always on the previous profile $F_{i-1}$ at point $A_{i}$ :

$$
F_{i}\left(A_{i}\right)=F_{i-1}\left(A_{i}\right) \quad \text { for } \quad i=2,3 \ldots n
$$

the profile of $\mathrm{F}_{\mathrm{i}}$ goes to zero at point $\mathrm{B}_{\mathrm{i}}$ :

$$
F_{i}\left(B_{i}\right)=0 \text { for } i=1,2, \ldots n
$$

and finally, the amount of the material added in $i$-th track is the same as in the first track. Therefore:

$$
\int_{A_{i}}^{B_{i}} F_{i} d x=\int_{A_{1}}^{B_{1}} F_{1} d x+\int_{A_{i}}^{B_{i-1}} F_{i-1} d x \text { for } i=1,2, \ldots n
$$

The right side of equation (4) represents the amount of new material added, plus material from previous track inside the overlap zone. Equations (2)-(4) are sufficient for model calculations when the functions $F_{i}$ are functions of the same type determined by 3 parameters.

The model is highly versatile with the possibility to substitute different functions. We have found parabolic function to be the most optimal as it has an excellent agreement with experimental results while still keeping the model very simple [9]. Our model always converges to a stable shape, i.e. a constant height and waviness is achieved after a few overlapped tracks, as would be physically 
expected. Due to the nature of coatings created using overlap of individual tracks the resulting surface has a wavy surface. Typically, for practical applications such a surface is not optimal and a flat surface is preferred with post-finishing machining $d$ after cladding.

Hence, we consider an effective coating thickness, which refers to the final thickness after machining and for practical purposes can be seen as the same as the thickness at the lowest point on a wavy surface. Therefore it is useful to consider comparative quantities, namely relative waviness and relative coating height. Relative surface waviness of the coating is defined in accordance with previous studies [10] as: $\left(\max \left(F_{i}(x)\right)-\min \left(F_{i}(x)\right)\right) / \max \left(F_{i}(x)(i \gg 1)\right)$. Relative coating height is the height of the coating measured from the substrate compared to the height of an individual track.

We have shown that in our model the final height and waviness do not depend on the $H / w$ ratio but depend only on the overlap ratio, $O R$. The model predictions using the parabolic functions resulted in an excellent prediction for an overall shape - the height and the waviness, of the profile of the laser clad coatings, Fig. 2.The clad was created using Colmonoy 33 powder on SS 304 substrate with $P=2200 \mathrm{~W}, S=20 \mathrm{~mm} / \mathrm{s}, F=346.7 \mathrm{mg} / \mathrm{s}$ and displacement $=1.9 \mathrm{~mm}$.

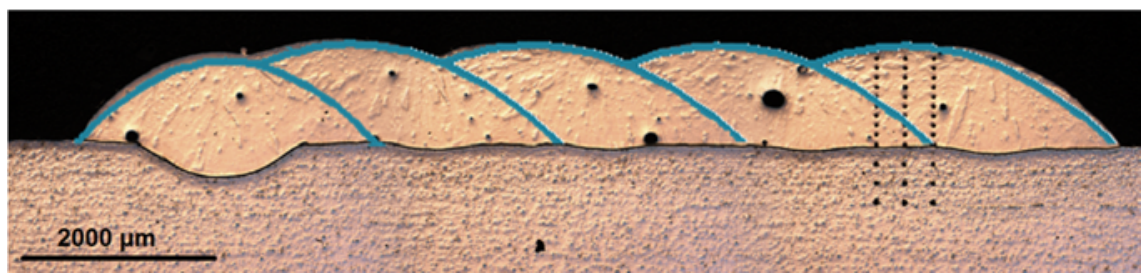

Figure 2: Comparison of experimentally observed profile of a single track and coating prepared by coaxial cladding and calculated profile (blue overlay) using parabolic shape function.

Data from a number of experiments conducted for several different ORs using different laser set-ups is compared to the model prediction for the parabolic function in Fig. 3. With the exception of the side cladding for a nozzle with a small opening the data fits the model prediction very well.

As a reasonable prediction can be attained solely based on the initial processing parameters that can be easily tuned in each experiment, the next step is a prediction of an entire coating geometry based on these parameters alone.

\section{Theoretical considerations}

Using numerical fits for exponential functions the relations between the relative coating height and waviness as a function of $O R$ predicted by the recursive model (Fig. 3) can be expressed as expressions (5) and (6) which is valid in the overlap ratio interval $0.0-0.8,[11]$. This gives expressions for relative coating height, $H_{r e l}$, and relative coating waviness, $W_{\text {rel }}$ : 


$$
\begin{array}{ll}
H_{r e l}=A e^{\frac{O R}{\beta}}+C & O R<0.8 \\
W_{r e l}=A e^{\frac{O R}{\beta}}+C & O R<0.8
\end{array}
$$

$A, \beta$ and $C$ are constants that were determined numerically and listed in Table 1. The values have been determined from the fits of the model predictions for parabolic function.

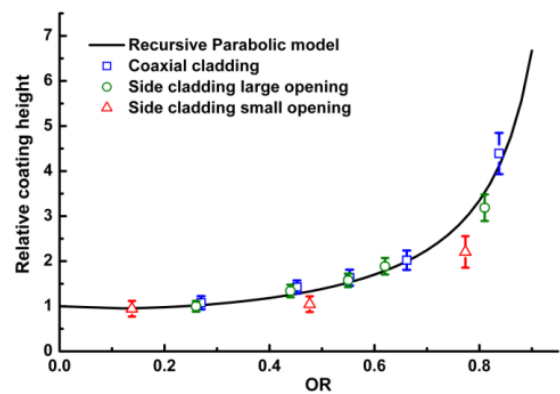

(a)

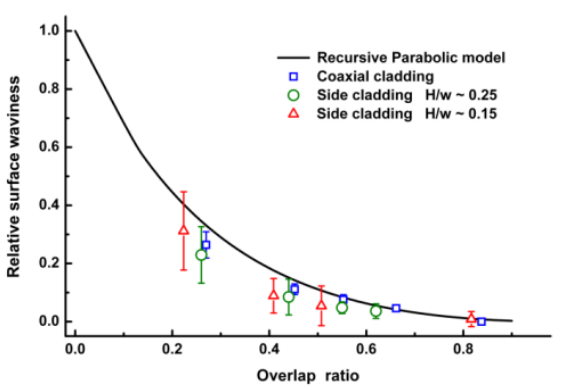

(b)

Figure 3: Parabolic function for the recursive model plotted against experimental data for relative coating height (a) and relative surface waviness (b) as a function of OR, [9].

Table 1: Values of constants in equations (5) and (6) for relative coating height and relative coating waviness for the example of the parabolic function, [11].

\begin{tabular}{|c|c|c|c|}
\hline & $A$ & $\beta$ & $C$ \\
\hline Height & 0.0454 & 0.205 & 0.862 \\
\hline Waviness & 1.051 & -0.253 & -0.0327 \\
\hline
\end{tabular}

We have shown $[2,3]$ that the clad height, $H$, depends only on the scanning speed, $S$, and the powder feeding rate $F$, leading to the following dependence:

$$
H=\alpha \frac{F}{S}+\gamma
$$

where $\alpha$ and $\gamma$ have dimensions $\left(\mathrm{m}^{2} / \mathrm{kg}\right)$ and $(\mathrm{m})$, respectively. Since the absolute value of the coating height, the height of the coating measured from the substrate, is a product of relative coating height and a single track height, $H$, we can combine equations (5), (6) and (7), to:

$$
H_{a b s}, W_{a b s}=a \frac{F}{S} e^{\frac{O R}{\beta}}+b e^{\frac{O R}{\beta}}+c \frac{F}{S}+d
$$

which is a function containing only constants $a, b, c, d$ and $\beta$, processing parameters, $F, S$, and an overlap ratio, $O R,[11]$. The relative coating height and the relative coating waviness only depend on the overlap ratio, i.e. $H_{\text {rel }}=f(O R)$ and $W_{\text {rel }}=f(O R)$.

The dimensions of the constants are $\left(\mathrm{m}^{2} / \mathrm{kg}\right)$ for $a$ and $c$ and $(m)$ for $b, d$ and $\beta$ is dimensionless. Equation (8) is true for both: absolute height, $H_{a b s}$, and absolute 
waviness, $W_{a b s}$, although the constants will be different in each case. The constants are determined from the aforementioned constants in equations (5), (6) and (7) once the equations are combined.

While equations (7) and (8) appear to show a convenient and simple description of the single track and coating geometry, the complete description is more complicated because the overlap ratio $O R$ is not a processing parameter. $O R$ depends on track width $w$ and on displacement, $D$, between successive tracks. Displacement refers to distance between points $A_{1}$ and $A_{2}$ in Fig. 1.

Previous experiments have shown that width of the laser track, $w$, depends linearly on laser power, $P$, and scanning speed, $S[2,3]$ :

$$
w=\delta\left(\frac{P}{\sqrt{S}}\right)+\omega
$$

where $\delta$ and $\omega$ have dimensions $\left(m^{1 / 2} W^{-1} s^{-1 / 2}\right)$ and $(m)$, respectively.

Therefore, both relative coating height and relative coating waviness can be predicted directly from experimental processing parameters. This then allows for a complete prediction of the geometry based purely on processing parameters. Equation (8) has been derived on the base of these statistical relations and therefore it also has this statistical meaning.

\section{Results and discussion}

\subsection{Model prediction}

Upon combining equations (7) and (9) with the recursive model it is possible to predict the coating profiles based on overlap of individual tracks from $P, S, F$ and displacement, $D$, between successive tracks. Fig. 4 shows results of the model predictions for a range of displacements and scanning speeds. The laser power and powder feeding rate are kept constant between individual plots for a more direct comparison, with $P=700 \mathrm{~W}$ and $F=120 \mathrm{mg} / \mathrm{s}$.

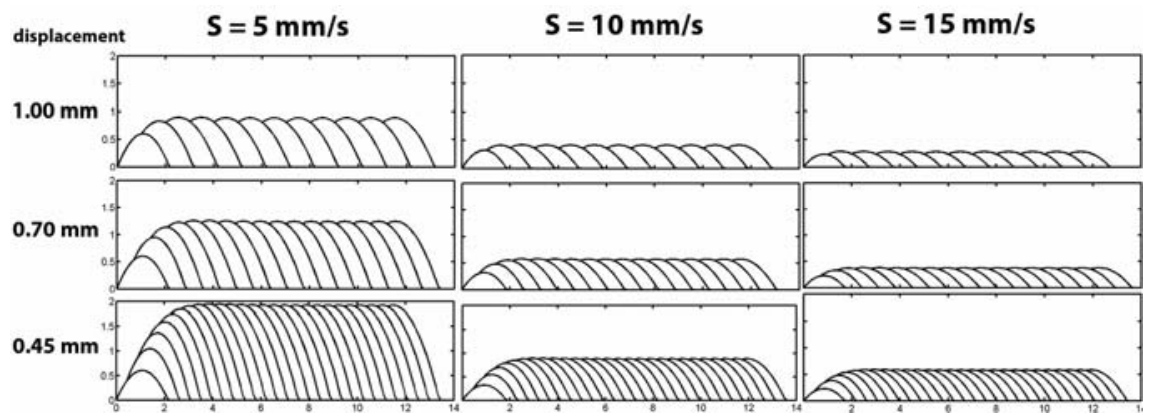

Figure 4: Profiles of coatings formed by overlap of individual tracks calculated for laser cladding with three different scanning speeds and three different laser track displacements. The units on the axes of all the plots are in $\mathrm{mm}$. 
In real experiments the height of the coating decreases with increasing scanning speed due the amount of powder acquired per unit length is less for a constant feeding rate and with increased overlap ratio, i.e. smaller displacement, the height grows. This is exactly what we predict in our model, Fig. 4. Furthermore, let us consider two more cases where we demonstrate model predictions for other sets of parameters. Fig. 5 shows a similar plot but for a range of feeding rates, with a different set of parameters kept constant; $P=1200 \mathrm{~W}$ and $S=10 \mathrm{~mm} / \mathrm{s}$.

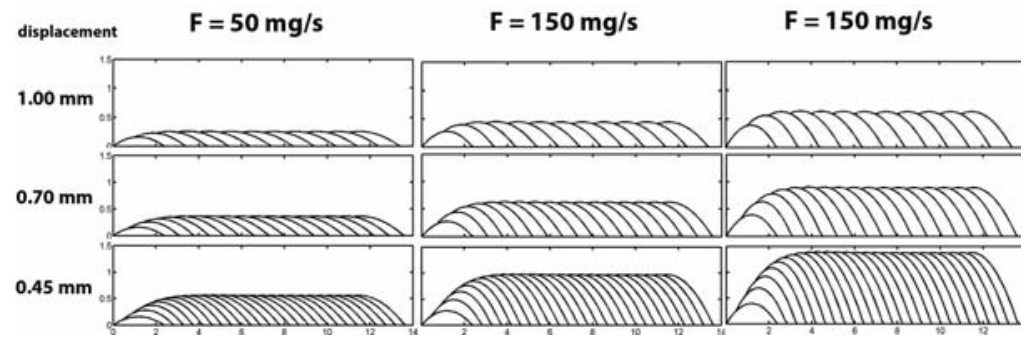

Figure 5: Profiles of coatings formed by overlap of individual tracks calculated for laser cladding with three different powder feeding rates and three different laser track displacements. The units on the axes of all the plots are in $\mathrm{mm}$.

Finally, Fig. 6 shows a plot for a range of laser powers with $S=10 \mathrm{~mm} / \mathrm{s}$ and $F=141.7 \mathrm{mg} / \mathrm{s}$. Looking at Figs $4-6$ it can be clearly seen how coating height and width relate to the processing parameters. The increase of feeding rate results purely in the increase of the coating height as it results from more material added while the width is kept the same as this is controlled by the laser power. In turn, the laser power increase increases merely the clad width as the beam follows a Gaussian distribution resulting in a wider track at higher powers. The scanning speed affects both the width and height as this has an effect the amount on both the energy delivered per unit area from the laser beam and also on the amount of material that is delivered in the given time window per unit length.

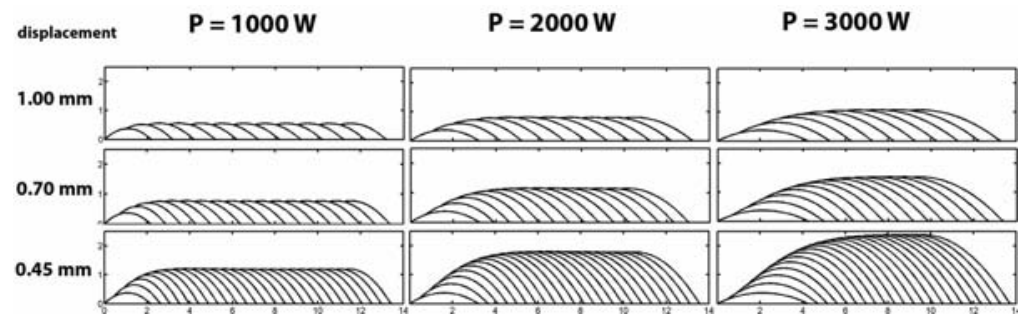

Figure 6: Profiles of coatings formed by overlap of individual tracks calculated for laser cladding with three different laser powers and three different laser track displacements. The units on the axes of all the plots are in $\mathrm{mm}$. 
Therefore, we demonstrate the possibility to predict the final geometry purely based on experimental inputs with very physically sound predictions.

\subsection{Experimental comparison}

In order to compare the predictions of our model to experiment we first need to obtain empirical constants based on the aforementioned combined parameters. To this end we have prepared many single tracks with different combinations of laser power, scanning speed and powder feeding rate which have resulted in different combinations of track width and height. The resulting clad heights and width were plotted against the controlling set of combined parameters, Fig. 7.
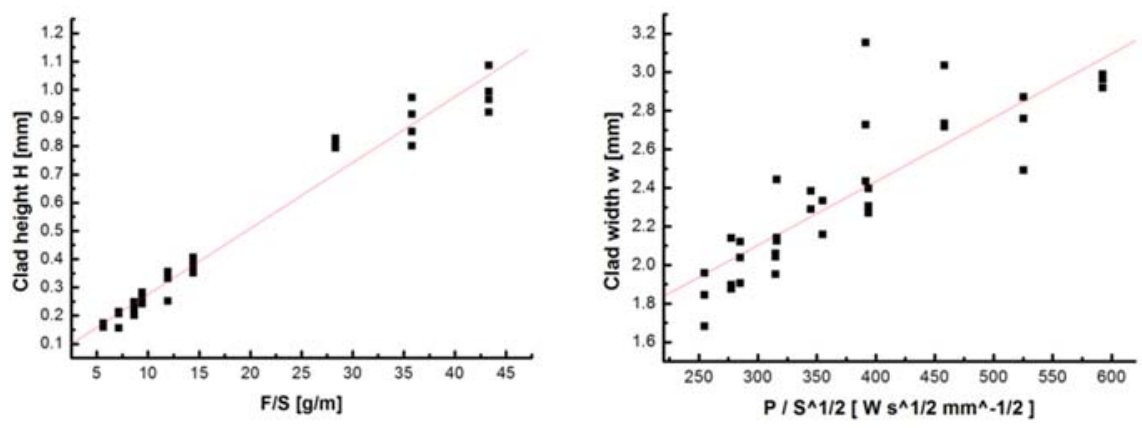

Figure 7: Plots of measured clad height and clad width against combined parameters with the straight line fits shown.

From these plots we are able to obtain the empirical constants via straight line fits. These laser clad experiments were done using Höganäs 3533-00 powder on SS 304 substrate and cladded using side cladding nozzle. The constants we have obtained are unique to the particular substrate, powder and to our laser set-up and these measurements need to be repeated if any of these is changed. The constants were:

$\begin{array}{llll}\alpha & \gamma & \delta & \omega \\ 0.02224 \mathrm{~m}^{2} / \mathrm{kg} & 0.37398 \mathrm{~m} & 0.00359 \mathrm{~m}^{1 / 2} \mathrm{~W}^{-1} \mathrm{~s}^{-1 / 2} & 1.0462 \mathrm{~m}\end{array}$

which refers to refers to the constants in equations (7) and (9).

Figs. 8-9 show cross sections of the final coating taken as polarized images in optical microscope with the model prediction overlay shown. The coatings were done with $P=1200 \mathrm{~W}, S=10 \mathrm{~mm} / \mathrm{s}, F=141.67 \mathrm{mg} / \mathrm{s}$ and displacement of 2.0 $\mathrm{mm}$ and $1.0 \mathrm{~mm}$, respectively.

The model shows a good agreement with experiments. In each case the starting and final position of each of the individual tracks as well as the whole coating matches the model prediction with an almost identical precision, with the height also having overall a good agreement and the character of the track shape is in some cases slightly off. The mismatch is mostly due to surface fluctuations 
during the solidification, surface tension and partly melted powder particles at the surface of the clad layers. These are not considered in the model.

This suggests that overall the model works very well despite the very simple assumptions. The model could be further improved by attempting to replace the simple parabolic function with other more complex functions. Comparable results were found with other displacements and with Colmonoy 33 powder.

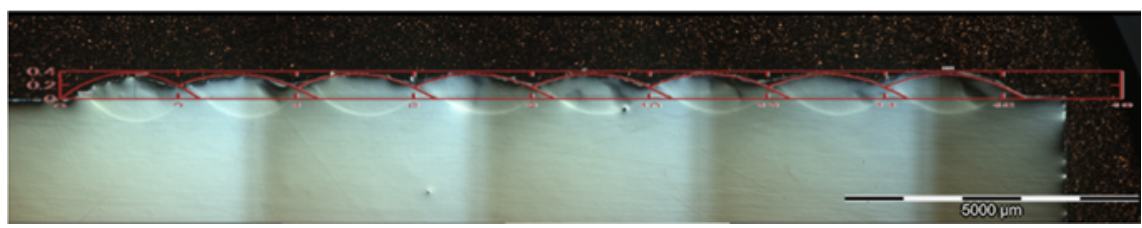

Figure 8: Comparison of a model prediction (red overlay) with 3533-00 coating on SS304 substrate, optical image. Displacement $=2 \mathrm{~mm}$.

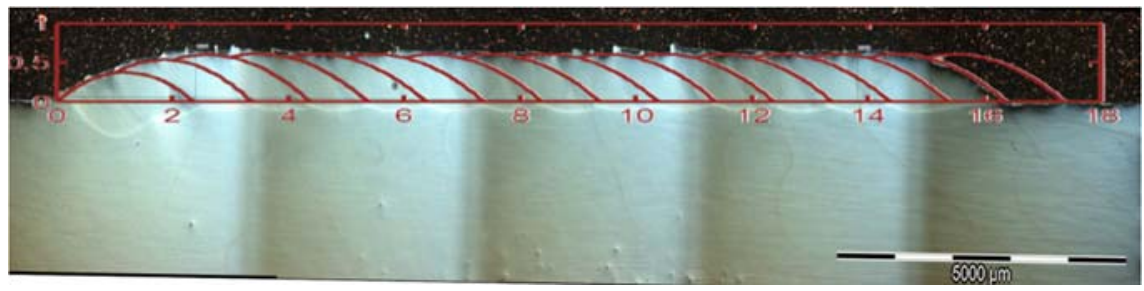

Figure 9: Comparison of a model prediction (red overlay) with 3533-00 coating on SS304 substrate, optical image. Displacement $=1 \mathrm{~mm}$.

The next step in our work is to extend these predictions to multi-layer coatings.

\section{Conclusions}

We have presented a very simple but very effective tool for the prediction of coating geometry for coatings formed by overlap of individual laser tracks in laser cladding. A full coating geometry can be predicted purely based on experimental inputs: scanning speed, laser power, powder feeding rate and displacement between successive tracks. The model contains experimental constants that depend on the particular set-up, which can be determined experimentally leading to a convenient prediction of the final laser clad geometry. The experimental comparison shows an excellent agreement with theoretical prediction for single layer coatings. 


\section{Acknowledgements}

This research was carried out under project number M41.7.12463 in the framework of the Research Program of the Materials innovation institute, M2i (www.m2i.nl). Sample shown in Fig. 2 was prepared by Ismail Hemmati.

\section{References}

[1] Toyserkani, E., Khajepour, A., \& Corbin, S., Laser Cladding. CRC Press, Boca Raton, 2005.

[2] de Oliveira, U., Ocelík, V., \& De Hosson, J.Th.M., Analysis of coaxial laser cladding processing conditions. Surface \& Coatings Technology 197, 127-136, 2005.

[3] Ocelík, V., de Oliveira, U., de Boer, M., \& De Hosson, J.Th.M., Thick Cobased coating on cast iron by side laser cladding: Analysis of processing conditions and coating properties. Surface \& Coatings Technology 201, 5875-5883, 2007.

[4] Felde, I., Reti., T., Zoltan., K., Costa, L., Colaco, R., Vilar, R., \& Vero, B., 2003. Proceedings from the $1^{\text {st }}$ International Surface Engineering Congress and the $13^{\text {th }}$ IFHTSE Congress, 7-10, Columbus, Ohio. ASM International, 237, October 2002.

[5] Kumar, A., \& Roy, S., Development of a theoretical process map for laser cladding using a three-dimensional conduction heat transfer model. Numer. Heat Transf. Part Appl. 56, 478-496. doi:10.1080/10407780903266489, 2009.

[6] Ni, L., Liu, J., Wu, Y., \& Yan, C., Optimization of laser cladding process variables based on neural network and particle swarm optimization algorithms. Zhongguo Jiguang/Chinese J. Lasers 38 . doi:10.3788/CJL20113802.0203003, 2011.

[7] Paulo Davim, J., Oliveira, C., \& Cardoso, A., Predicting the geometric form of clad in laser cladding by powder using multiple regression analysis (MRA). Mater. Des. 29, 554-557. doi:10.1016/j.matdes.2007.01.023, 2008.

[8] Liu, H., Yu, G., He, X., Li, S., Zheng, C., Ning, W., \& Ge, Z., Threedimensional numerical simulation of transient temperature field and coating geometry in powder feeding laser cladding. Zhongguo Jiguang Chinese J. Lasers 40. doi:10.3788/CJL201340.1203007, 2013.

[9] Ocelík, V., Nenadl, O., Palavra, A., \& De Hosson, J.Th.M., On the geometry of coating layers formed by overlap. Surface \& Coatings Technology 242, 54-61, 2014.

[10] Paul, C.P., Mishra, S.K., Kumar, A., \& Kukreja, L.M., Laser rapid manufacturing on vertical surfaces: Analytical and experimental studies. Surf. Coat. Technol. 224, 18, 2014.

[11] Nenadl O., Ocelík, V., Palavra, A., \& De Hosson, J.Th.M., The prediction of coating geometry from main processing parameters in laser cladding. Physics Procedia 56 220-227, 2014. 